\title{
COMPARATIVO ENTRE BIODIESEL DE DENDÊ E TUCUMÃ NO DESEMPENHO OPERACIONAL DE TRATOR AGRÍCOLA
}

\author{
Leomar Paulo de Lima ${ }^{1}$, Afonso Lopes ${ }^{2}$, Melina Cais Jejcic de Oliveira ${ }^{3}$, Murilo Coelho Teodoro Neves ${ }^{4}$, \\ Gilberto Hirotsugu Azevedo Koike ${ }^{5}$
}

\begin{abstract}
RESUMO
A viabilidade do biodiesel como fonte alternativa de energia depende de vários fatores, entre os quais se destacam a proporção de mistura do biodiesel ao diesel e incentivo de uma organização da sua cadeia produtiva, de modo a contemplar as dimensões econômicas, sociais e ambientais. O objetivo do trabalho foi comparar biodiesel de dendê e tucumã no desempenho operacional de trator agrícola. O experimento foi realizado no Departamento de Engenharia Rural da FCAV-UNESP, Jaboticabal, SP, utilizando-se um trator 4x2 TDA com potência máxima no motor de $80,9 \mathrm{~kW}(110 \mathrm{cv})$. O delineamento experimental foi inteiramente casualizado em esquema fatorial $6 \times 2$, em que as combinações dos fatores foram seis proporções de mistura biodiesel/diesel (B0, B5, B25, B50, B75 e B100, em que o número indica a percentagem de biodiesel no diesel) e dois tipos de biodiesel (dendê e tucumã). Avaliaram-se potência média de tração na barra ( $\mathrm{kW}$ ), rotação do motor (rpm), consumo volumétrico $\left(\mathrm{L} \mathrm{h}^{-1}\right)$, consumo ponderal $\left(\mathrm{kg} \mathrm{h}^{-1}\right)$, consumo específico $\left(\mathrm{g} \mathrm{kWh}^{-1}\right)$ e opacidade da fumaça. Os resultados evidenciaram que a partir do uso da mistura com 50\% de biodiesel ocorreu aumento no consumo específico, entretanto o tipo de biodiesel não influenciou a referida variável. A opacidade da fumaça reduziu com o incremento das proporções de mistura de biodiesel no diesel, destacando que o tipo de biodiesel também influenciou esta medida, sendo o tucumã de menor opacidade.
\end{abstract}

Palavras-Chave: biocombustível, força de tração, consumo específico.

\section{ABSTRACT \\ COMPARATIVE OPERATIONAL PERFORMANCE OF AGRICULTURAL TRACTOR RUN ON BIODIESEL FROM DENDE AND BRAZILIAN PALM}

The feasibility of biodiesel as an alternative fuel depends on several factors, such as proportion of biodiesel in diesel and the encouragement of organizations related to production chain, in a way that contemplates economic, social and environmental aspects. This study was done to compare operational performance of farm tractors running on biodiesel from dende oil or Brazilian palm oil. The experiment was conducted at the Department of Agricultural Engineering, FCAV-Jaboticabal, SP, using a $4 \times 2$ TDA tractor with maximum engine power of $80.9 \mathrm{~kW}(110 \mathrm{hp})$. The experiment was done in completely randomized design in a $6 \times 3$ factorial, in which the factors were represented by proportions of biodiesel/diesel mix (B0, B5, B25, B50, B75 and B100, where the number indicates the percentage of biodiesel in diesel) and types of biodiesel (dende or Brazilian palm). We evaluated average traction power in the bar $(\mathrm{kW})$, engine rotation (rpm), volumetric consumption $\left(\mathrm{L} \mathrm{h}^{-1}\right)$, consumption by weight $\left(\mathrm{kg} \mathrm{h}^{-1}\right)$, specific consumption $\left(\mathrm{g} \mathrm{kWh}^{-1}\right)$ and smoke opacity. The results showed that specific consumption increased when 50\% biodiesel mix was used, but the type of biodiesel had no influence. The smoke opacity decreased with increasing proportions of biodiesel in the fuel, and the smoke opacity was still less with the Brazilian palm biodiesel.

Keywords: Biofuel, traction force, specific consumption

\section{Recebido para publicação em 23/01/2012. Aprovado em 21/03/2012.}

1- Prof. (Licenciatura em Ciências Agrárias) e Doutorando em Agronomia - DER/FCAV-UNESP, Jaboticabal (SP), leomaragro@yahoo.com.br

2- Prof. Adjunto, Departamento de Engenharia Rural - DER/FCAV-UNESP, Jaboticabal (SP), Afonso@fcav.unesp.br

3- Eng. Agrônoma e Doutoranda em Agronomia - DER/FCAV-UNESP, Jaboticabal (SP), melina_cais@yahoo.com.br

4- Eng. Agrônomo e Mestrando em Agronomia - DER/FCAV-UNESP, Jaboticabal (SP), murilocoelho.tn@gmail.com

5- Eng. Mecânico Doutor (Consultor Autônomo), gkoike@bol.com.br

\section{REVENG}




\section{INTRODUÇÃO}

Historicamente, o uso de óleos vegetais como combustível teve início com o desenvolvimento dos motores diesel no final do século XIX por Rudolph Diesel (PINTO et al., 2005). Todavia, no início do século XX, o diesel passou a ser o combustível utilizado para esse tipo de motor devido ao seu baixo custo e por razões técnicas.

Durante as duas crises do petróleo ocorridas nas décadas de 30 e 70, foi retomada a ideia do uso de óleos vegetais como combustível; entretanto, a mesma não obteve sucesso (SUAREZ et al., 2007). Além disso, houve uma maior preocupação em atender à crescente demanda energética mundial, o que, consequentemente, levou ao crescimento da consciência ambiental devido às mudanças climáticas associadas à liberação de gases da queima de combustíveis fósseis. Todos esses fatores impulsionaram a busca por combustíveis de fontes renováveis, os quais voltaram a ser fortemente discutidos e pesquisados. (PIMENTEL et al., 2006; LÔBO; FERREIRA, 2009).

Para produzir combustíveis alternativos, o Brasil possui diversas espécies de oleaginosas. No entanto, o dendê apresenta-se como oleaginosa de grande significado comercial, sendo capaz de produzir grande quantidade de óleo por hectare, podendo atingir até $5000 \mathrm{~kg}$ de óleo por hectare por ano, além de possuir menor custo de produção, quando comparado com outras oleaginosas (SANTANA et al., 2005). O tucumã é uma das várias espécies oleaginosas amazônicas que satisfazem os critérios fundamentais para o uso adequado e sustentável de espécies oleaginosas nativas na produção de biodiesel na Amazônia (FIGLIUOLO et al., 2007).

O biodiesel é perfeitamente miscível ao óleo diesel, podendo ser utilizado puro ou em misturas sem que qualquer adaptação nos motores seja necessária. As misturas binárias de biodiesel e óleo diesel são designadas pela abreviação $\mathrm{B} X$, onde $X$ é a percentagem de biodiesel adicionada à mistura e o B é a primeira letra de mistura em inglês (PARENTE, 2003).

Figliuolo et al. $(2004,2007)$ estudando o potencial das amêndoas de tucumã para a produção de biodiesel, obtiveram valores de lipídeos totais variando de 40 a $50 \%$, sendo que sua proporção no fruto foi de $36,9 \%$, valor próximo ao obtido por Nascimento e Dallan (2007), de 39,7\%, e por Castro et al. (2006) que, estudando o potencial dos óleos, de algumas espécies de palmeiras da Amazônia, para produção de biodiesel, obtiveram $36 \%$ de óleo na amêndoa de tucumã, conseguindo dessa forma a extração do óleo das amêndoas por prensagem mecânica.

Estudos para avaliar o desempenho operacional de motores utilizando Biodiesel não apontaram indícios de aumento de desgaste, comprometimento de potência ou redução na durabilidade, no entanto vários autores observaram aumento no consumo de combustível à medida que se aumenta a proporção de uso do biocombustível. Comparando B0 com B100, Lopes et al. (2004), ensaiando trator agrícola, verificaram que o consumo de combustível específico aumentou $11 \%$, entretanto não foram observadas anomalias no funcionamento. Camara (2009), em experimento similar, evidenciou que, a partir de B25, houve aumento no consumo específico, chegando a $14 \%$, relacionando B0 com B 100 . Na mesma linha de pesquisa, Grotta et al. (2008) verificaram que o consumo de combustível horário aumentou 4,9\%, o consumo ponderal $13,0 \%$ e o consumo específico $11,4 \%$, confrontando B0 com B100.

Apesar de percebida há muito tempo, foi principalmente na $2^{\mathrm{a}}$ metade do século XX que a poluição do ar assumiu destaque entre a população e junto à comunidade técnico-científica.

Para verificar a concentração de material particulado presente na fumaça, utiliza-se a opacidade da fumaça, que indica a cor dessa, normalmente uma fumaça muito escura (preta) é sinal de excesso de combustível (mistura rica), branca usualmente composta de vapor d'água condensada e combustível líquido não queimado e azul composta por gotículas resultantes da combustão incompleta de combustível e/ou lubrificante (TECNOMOTOR, 2010), sendo que quanto mais escura for a fumaça, maiores serão a opacidade e a quantidade de material particulado.

O opacímetro determina a opacidade da fumaça do gás eliminado, e este aparelho coleta somente uma parte do fluxo de gás, através de um tubo de captação e uma sonda colocados no cano de escape do veículo. Verificou-se que, utilizando-se de biodiesel de dendê, ocorreu redução de 53\% na 
opacidade da fumaça quando comparado com o diesel (CAMARA et al., 2007).

Pressupõe-se que misturas com porcentagens maiores de biodiesel aumentem o consumo de combustível e reduzam a opacidade da fumaça, e que os diferentes tipos de biodiesel mantenham estável o funcionamento do motor, pouco influenciando no consumo de combustível e na opacidade da fumaça. Objetivou-se com este trabalho, comparar biodiesel de dendê e tucumã no desempenho operacional de trator agrícola.

\section{MATERIAL E MÉTODOS}

O trabalho foi conduzido em área do Laboratório de Máquinas e Mecanização Agrícola - LAMMA, do Departamento de Engenharia Rural, da Universidade Estadual Paulista - UNESP, Campus de Jaboticabal. Aárea está situada lateralmente à Via de Acesso Prof. Paulo Donato Castellane, km 5, possui localização geodésica definida pelas coordenadas $21^{\circ} 15^{\prime}$ latitude sul e $48^{\circ} 18^{\prime}$ longitude oeste, com altitude média de $570 \mathrm{~m}$. A região apresenta temperatura média anual de $22,2{ }^{\circ} \mathrm{C}$, precipitação média anual de 1.425 mm, umidade relativa média de $71 \%$ e pressão atmosférica de $94,3 \mathrm{kPa}$. O clima da região, segundo a classificação de Köeppen, é do tipo Aw, definido como tropical úmido, com estação chuvosa no verão e seca no inverno.

O solo da área experimental foi classificado como Latossolo Vermelho Eutroférrico típico, com relevo suave ondulado e declividade média de $3 \%$, conforme o Sistema Brasileiro de Classificação de Solos (ANDREOLI; CENTURION, 1999). O teor médio de água no período do ensaio, no perfil de 0 a 15 e 15 a $0,30 \mathrm{~cm}$ de profundidade, foi 11,2 e $13,4 \%$, respectivamente, segundo o método gravimétrico-padrão. A análise granulométrica da camada de $0-20 \mathrm{~cm}$ para argila, silte, areia fina e areia grossa foi 51;29; 10 e 10\%, respectivamente, sendo o mesmo considerado como textura argilosa.

Os tipos de biodiesel utilizados foram do tipo dendê (Elaesis guineensis) e tucumã (Astrocaryum aculeatum). O processo de produção e o fornecimento dos biocombustíveis ficaram a cargo do Laboratório de Desenvolvimento de Tecnologias Limpas - LADETEL, da Universidade de São Paulo - USP, Campus de Ribeirão Preto.
O biodiesel ensaiado foi produzido de acordo com as especificações da Resolução ANP $n^{\circ}$ 4, de 2 de fevereiro de 2010 (BRASIL, 2010).

Utilizou-se um trator de teste da marca Valtra, modelo BM110, 4x2 com tração dianteira auxiliar (TDA), potência máxima no motor de $80,9 \mathrm{~kW}$ $(110 \mathrm{cv})$, massa total de $6.160 \mathrm{~kg}$, distribuídos 40 e $60 \%$ nos eixos dianteiro e traseiro, respectivamente, equipado com pneus 14.9-24 no eixo dianteiro e 23.1-26 no eixo traseiro, sendo tal trator instrumentado para a realização do teste. No momento dos ensaios, o trator operou a 2100 rpm, sendo que a velocidade média de trabalho conseguida foi de $5,3 \mathrm{~km} \mathrm{~h}^{-1}$ com a combinação do câmbio $3^{\mathrm{a}} \mathrm{L}$.

Para oferecer carga na barra de tração foi utilizado um trator de frenagem da marca Valtra, modelo BH140, 4x2, com tração dianteira auxiliar (TDA), potência máxima no motor de $103 \mathrm{~kW}(140$ cv), massa total de $7.400 \mathrm{~kg}$, distribuídos 40 e $60 \%$ nos eixos dianteiro e traseiro, respectivamente, equipado com pneus 14.9-24 no eixo dianteiro e 23.1-26 no eixo traseiro.

$\mathrm{O}$ trator de frenagem foi utilizado desligado e engrenado, pois a única função era oferecer carga na barra de tração do trator de teste o mais uniforme possível, sendo que a velocidade média de trabalho conseguida foi de $5,3 \mathrm{~km} \mathrm{~h}^{-1}$ com a combinação do câmbio $4^{\mathrm{a}} \mathrm{L}$ e acoplado ao trator de teste por meio de um cabo de aço, formando um comboio. Um ensaio preliminar, também denominado de ensaio-piloto, foi utilizado com a finalidade de definir a carga correspondente ao esforço máximo, tecnicamente viável, que o trator de teste poderia tracionar, obtendo-se um resultado de aproximadamente $25 \mathrm{kN}$.

Para determinar a força de tração na barra, utilizou-se de célula de carga (M.SHIMIZU, modelo TF400), com capacidade máxima de medição de $100 \mathrm{kN}$. Para medir o consumo de combustível, utilizou-se de um protótipo com dois medidores de fluxos instalados em série com dois medidores de temperatura. A velocidade real de deslocamento foi obtida por meio de radar (modelo RVS II). Todos os sensores enviaram os sinais a um sistema de aquisição de dados (micrologger CR23X, sendo o mesmo programado para obter dados numa frequência de 10 leituras por segundo. 
$\mathrm{O}$ protótipo utilizado para determinar o consumo em todas as parcelas forneceu valores expressos em $\mathrm{mL} \mathrm{s}^{-1}$, referentes ao débito da bomba injetora e o retorno dos bicos, respectivamente. Por meio de diferença entre os valores, obteve-se o volume realmente consumido pelo trator durante $o$ percurso da parcela.

O delineamento experimental foi inteiramente casualizado, esquema fatorial $6 \times 2$, com quatro repetições, totalizando 12 tratamentos e 48 observações. As combinações dos fatores foram seis proporções de mistura biodiesel/diesel (B0, B5, B25, B50, B75 e B100, em que o número indica a percentagem de biodiesel no diesel) e dois tipos de biodiesel (dendê e tucumã). Ressaltase que para o ensaio dinâmico, cada parcela experimental possuía $30 \mathrm{~m}$ de comprimento e, entre si, na direção longitudinal, foi reservado espaço de $15 \mathrm{~m}$ destinado à realização de manobras, tráfego de máquinas e estabilização do conjunto motomecanizado.

Para a obtenção do consumo horário expresso em massa, foi necessário determinar a densidade em função da temperatura nas seis proporções de mistura. A temperatura do combustível foi monitorada nos mesmos pontos onde foram realizadas as medidas de fluxo. A densidade foi determinada no intervalo de temperatura de 10 a 70 ${ }^{\circ} \mathrm{C}$, em passos de 5 e $5{ }^{\circ} \mathrm{C}$, pela análise de regressão, obtida por meio de análise estatística.

De posse desses dados, determinaram-se o consumo horário (volumétrico e ponderal) e o consumo específico. O consumo horário volumétrico foi medido com base no volume consumido e no tempo de percurso em cada parcela, conforme a Equação 1:

$$
C h v=\frac{V a-V r}{t} 3,6
$$

em que

Chv $=$ consumo horário volumétrico $\left(\mathrm{L} \mathrm{h}^{-1}\right)$;

$\mathrm{Va}=$ volume do combustível de alimentação $(\mathrm{mL})$; $\mathrm{Vr}=$ volume do combustível de retorno $(\mathrm{mL}) ; \mathrm{e}$ $\mathrm{t}=$ tempo de percurso na parcela(s).

Para o cálculo do consumo horário ponderal, considerou-se a influência da densidade do combustível de alimentação e de retorno no momento do teste, conforme equação 2 :

$$
C h p=\frac{(V a D c a-V r D c r)}{t} 0,0036
$$

em que

Chp $=$ consumo horário ponderal $\left(\mathrm{kg} \mathrm{h}^{-1}\right)$;

$\mathrm{Va}=$ volume do combustível de alimentação $(\mathrm{mL})$; Dca $=$ densidade do combustível de alimentação $\left(\mathrm{kg} \mathrm{m}^{-3}\right)$;

$\mathrm{Vr}=$ volume do combustível de retorno $(\mathrm{mL})$;

Dcr $=$ densidade do combustível de retorno $\left(\mathrm{kg} \mathrm{m}^{-3}\right)$; e

$\mathrm{t}=$ tempo de percurso na parcela $(\mathrm{s})$.

O consumo específico é o consumo de combustível expresso em unidade de massa por unidade de potência requerida na barra de tração, conforme equação 3 :

$$
\mathrm{Ce}=\left(\frac{\mathrm{Chp}}{\mathrm{PB}}\right) 1000
$$

em que

$\mathrm{Ce}=$ consumo específico $\left(\mathrm{g} \mathrm{kW} \mathrm{h}^{-1}\right)$;

$\mathrm{Chp}=$ consumo horário ponderal $\left(\mathrm{kg} \mathrm{h}^{-1}\right)$; e

$\mathrm{PB}=$ potência na barra de tração $(\mathrm{kW})$.

Os ensaios referentes à opacidade da fumaça do cano de escape do trator foram realizados de acordo com o método da aceleração livre, que é o regime de rotação a que o motor é submetido com o acelerador no seu curso máximo, sendo a potência desenvolvida absorvida somente pela inércia dos componentes mecânicos do motor (embreagem, árvore-piloto da caixa de mudanças), uma vez que o veículo está estacionado. As medições de opacidade são feitas em K, que é o coeficiente de absorção de luz, e tem como unidade $\mathrm{m}^{-1}$ (TECNOMOTOR, 2010). Ao término de cada determinação, realizou-se a drenagem completa do sistema de alimentação, evitando, com isso, a contaminação do ensaio seguinte. Além disso, depois de trocado o combustível, o motor ficou em funcionamento 
em torno de dez minutos antes do início de cada ensaio para estabilizar as medições.

Os dados foram tabulados e submetidos à análise de variância e ao teste de comparação de médias de Tukey, a $5 \%$ de probabilidade, conforme recomendação de Banzatto e Kronka (2006).

Para a opacidade da fumaça e consumo específico de combustível foi estudado modelo de ajuste de regressão que melhor explicasse o comportamento dessas variáveis, em função da proporção de biodiesel. Para o caso da densidade, ajustou-se um modelo de superfície de resposta que também explicasse essa variável em função da temperatura e da proporção de biodiesel.

Neste trabalho, utilizou-se da análise de variância (teste F) para selecionar o modelo de equação de maior expoente significativo.

\section{RESULTADOS E DISCUSSÃO}

Os resultados de cada variável foram discutidos em tópicos separados para facilitar o entendimento. No Quadro 1 encontra-se a síntese da análise de variância e teste de médias, para as variáveis potência na barra, rotação no motor, consumo horário volumétrico, ponderal e específico.

Os fatores tipo e proporção de biodiesel não influenciaram a potência na barra de tração, que apresentou média de $33,74 \mathrm{~kW}$. Esse comportamento é explicado em função da potência na barra de tração ser o produto da força, mantida próxima a $23 \mathrm{kN}$, com a velocidade que, neste experimento também não apresentou variação significativa. Tais resultados concordam com Lopes (2006), que trabalhou com várias fontes e processos da rota de produção de biodiesel no desempenho de um trator com potência média na barra de aproximadamente $32,0 \mathrm{~kW}$. Estes resultados reforçam que o uso de biodiesel não afeta a potência disponível na barra de tração, pois a forma de compensação do menor poder calorífico do biodiesel é o aumento no consumo de combustível para que a potência média na barra de tração não venha ser comprometida.

O tipo de biodiesel não influenciou na rotação do motor. No fator proporção de mistura, visualiza-se que B0, B5, B25 e B50 apresentaram valores maiores que a B100, ocorrendo queda na rotação do motor a partir

Quadro 1. Síntese da análise de variância e teste de médias para as variáveis potência média na barra (PB), rotação do motor $(\mathrm{RM})$, consumo horário volumétrico $(\mathrm{Chv})$, consumo horário ponderal (Chp) e consumo específico $(\mathrm{Ce})$

\begin{tabular}{|c|c|c|c|c|c|}
\hline Fatores & $\begin{array}{l}\text { PB } \\
\mathrm{kW}\end{array}$ & $\begin{array}{l}\mathbf{R M} \\
\mathrm{rpm}\end{array}$ & $\begin{array}{l}\text { Chv } \\
\mathrm{L} \mathrm{h}^{-1}\end{array}$ & $\begin{array}{c}\text { Chp } \\
\mathrm{kg} \mathrm{h}^{-1}\end{array}$ & $\begin{array}{c}\mathbf{C e} \\
\mathrm{g} \mathrm{kWh}^{-1}\end{array}$ \\
\hline \multicolumn{6}{|c|}{ Tipo de Biodiesel (TB) } \\
\hline Dendê & $33,61 \mathrm{a}$ & $1888,50 \mathrm{a}$ & $14,19 \mathrm{a}$ & $12,29 \mathrm{a}$ & $367,28 \mathrm{a}$ \\
\hline Tucumã & $33,88 \mathrm{a}$ & $1889,29 \mathrm{a}$ & $14,09 \mathrm{a}$ & $12,16 \mathrm{a}$ & $363,55 \mathrm{a}$ \\
\hline \multicolumn{6}{|c|}{ Proporção de Biodiesel (Bn) (\%) } \\
\hline 0 & 33,61 a & $1915,37 \mathrm{a}$ & $12,74 \mathrm{a}$ & $10,87 \mathrm{a}$ & $336,28 \mathrm{a}$ \\
\hline 5 & $33,97 \mathrm{a}$ & 1911,62 b & $13,21 \mathrm{~b}$ & $11,50 \mathrm{ab}$ & 339,16 a \\
\hline 25 & $33,74 \mathrm{a}$ & $1895,37 \mathrm{bc}$ & $13,27 \mathrm{~b}$ & $11,20 \mathrm{~b}$ & $332,72 \mathrm{a}$ \\
\hline 50 & $34,01 \mathrm{a}$ & $1886,25 \mathrm{c}$ & $14,43 \mathrm{c}$ & $12,55 \mathrm{c}$ & $370,27 \mathrm{~b}$ \\
\hline 75 & $33,90 \mathrm{a}$ & $1878,12 \mathrm{~d}$ & $15,49 \mathrm{~d}$ & $13,48 \mathrm{~d}$ & $398,70 \mathrm{c}$ \\
\hline 100 & $33,25 \mathrm{a}$ & $1846,62 \mathrm{~d}$ & $15,69 \mathrm{~d}$ & $13,76 \mathrm{~d}$ & $415,36 \mathrm{~d}$ \\
\hline \multicolumn{6}{|l|}{ TESTE $F$} \\
\hline $\mathrm{TB}$ & 2,3317 Ns & 0,1113 NS & $1,6863^{\mathrm{NS}}$ & \multirow{2}{*}{$\begin{array}{c}1,5766^{\mathrm{NS}} \\
100,5336 \\
* *\end{array}$} & 1,4204 NS \\
\hline $\mathrm{B}_{\mathrm{n}}$ & $1,6137^{\mathrm{NS}}$ & $75,0458 * *$ & $192,7059 * *$ & & $84,6786 * *$ \\
\hline $\mathrm{TBxB}_{n}$ & $2,3586 \mathrm{NS}$ & $0,5730 \mathrm{Ns}$ & $0,5807^{\mathrm{NS}}$ & $1,1058 \mathrm{NS}$ & $1,4801 \mathrm{NS}$ \\
\hline MEDIA & 33,74 & 1888,89 & 14,14 & 12,23 & 365,42 \\
\hline C.V. $(\%)$ & 1,86 & 0,43 & 1,80 & 2,81 & 2,96 \\
\hline
\end{tabular}

Médias seguidas pela mesma letra minúscula na coluna, não diferem entre si, pelo teste de Tukey, a $5 \%$ de probabilidade. **: significativo $(\mathrm{P}<0,01)$; *: significativo $(\mathrm{P}<0,05)$; NS: não significativo; C.V.: coeficiente de variação. 
de B5. A rotação do motor foi $68,75 \mathrm{rpm}$ menor quando se utilizou biodiesel B100, redução esta de $3,58 \%$ quando comparada com o diesel (B0). Esse comportamento pode ser explicado em função do menor poder calorífico do biodiesel em relação ao diesel. Resultados semelhantes foram encontrados por Camara (2009).

A variável consumo volumétrico de combustível não foi influenciada pelo tipo de biodiesel. Porém, em relação à proporção de biodiesel, ao comparar B0 e B100, verificase que o consumo aumentou $23,15 \%$. Esse aumento é função do menor poder calorífico do biodiesel em relação ao diesel, sendo necessário queimar mais combustível para realizar a mesma quantidade de trabalho. Esses resultados condizem com os encontrados por Tabile et al. (2009) e Murugesan et al. (2009), ressaltando-se que o consumo volumétrico é a informação mais utilizada no meio dos agricultores.

O consumo ponderal teve comportamento semelhante ao volumétrico. A relevância dessa medida é, para os profissionais que trabalham na distribuição de combustíveis, com a quantidade de massa que sai da origem sendo a mesma que chega ao destino final. Ademais, serve também para acompanhar a qualidade do produto que considera-se a densidade.

$\mathrm{O}$ tipo de biodiesel não influenciou o consumo específico de combustível. Com relação às proporções de mistura de biodiesel no diesel, nota-se que a partir de B50 ocorreu diferença significativa para esta variável. Todavia, comparando B0 a B100, o consumo específico aumentou 26,34\%. Esse aumento se deve ao menor poder calorífico do biodiesel em relação ao diesel. Diferentes valores para a variação do consumo específico de combustível são observados por outros autores Castellanelli et al. (2008) e Corrêa et al. (2008). O consumo específico é a forma mais utilizada no meio científico para comparar tratamentos, pois leva em consideração a quantidade de combustível consumida, potência desenvolvida e densidade do produto.

O comportamento do consumo específico em função da proporção de mistura teve comportamento linear, conforme se nota na Figura 1.

Os resultados de opacidade da fumaça do trator foram apresentados na forma de Equação, Figura e Quadro. No Quadro 2 observa-se que a interação entre os fatores tipo e proporção de biodiesel foi significativa. Analisando-se o fator tipo de biodiesel (na linha, Quadro 3), verificase que a opacidade da fumaça reduziu a medida que aumentou a quantidade de biodiesel na mistura, destacando-se o biodiesel de tucumã com uma redução de $60 \%$ e dendê $36,25 \%$, respectivamente. Estes resultados reforçam os relatos de Dabdoub et al. (2009) de que, as propriedades do biodiesel são fortemente

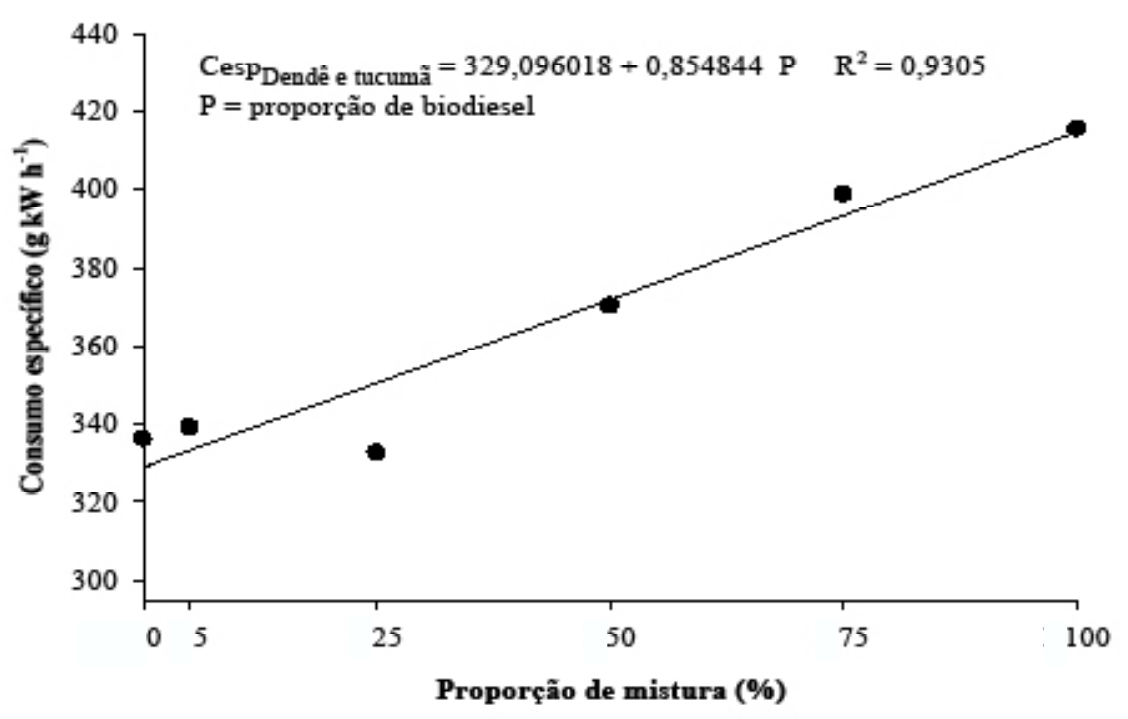

Figura 1. Modelo de regressão para consumo específico (Cesp) em função da proporção de mistura de biodiesel de dendê e tucumã no diesel. 
influenciadas pelas características individuais dos ésteres graxos que formam esse combustível. Peres et al. (2007) caracterizaram e determinaram poder calorífico e número de cetano de vários tipos de biodiesel através da cromatografia, os autores observaram que a propriedade número de cetano pode variar entre os diferentes tipos de biodiesel, podendo influenciar nas emissões do motor.

Analisando-se a variável proporção (na coluna), observa-se no Quadro 3 que, em todas as proporções de mistura, o biodiesel de tucumã, apresentou menor opacidade da fumaça em relação ao biodiesel de dendê. Esta redução na opacidade é devido a menor emissão de material particulado pelo uso do biodiesel. Os resultados do presente trabalho condizem com os encontrados por Lopes et al. (2007) e Tabile et al. (2009).

O comportamento da opacidade da fumaça em função da proporção de mistura para os três tipos de biodiesel teve comportamento linear, conforme se nota na Figura 2.

Quadro 2. Síntese da análise de variância e teste de médias para a variável opacidade da fumaça

\begin{tabular}{cc}
\hline Fatores & Opacidade \\
\hline Tipo de Biodiesel (TB) & $\left(\mathrm{m}^{-1}\right)$ \\
\hline Dendê & 1,38 \\
Tucumã & 1,03 \\
\hline Proporção de biodiesel (Bn) (\%) & 1,53 \\
5 & 1,44 \\
25 & 1,29 \\
50 & 1,15 \\
75 & 1,02 \\
100 & 0,81 \\
\hline TESTE F & $1663,7583 * *$ \\
TB & $662,5644 * *$ \\
$B_{\mathrm{n}}$ & $45,2125 * *$ \\
TBxB & 4,20 \\
\hline C.V. $(\%)$ & 1,21
\end{tabular}

Médias seguidas pela mesma letra minúscula na coluna, não diferem entre si, pelo teste de Tukey, a $5 \%$ de probabilidade. **: significativo $(\mathrm{P}<0,01)$; *: significativo $(\mathrm{P}<0,05)$; NS: não significativo; C.V.: coeficiente de variação

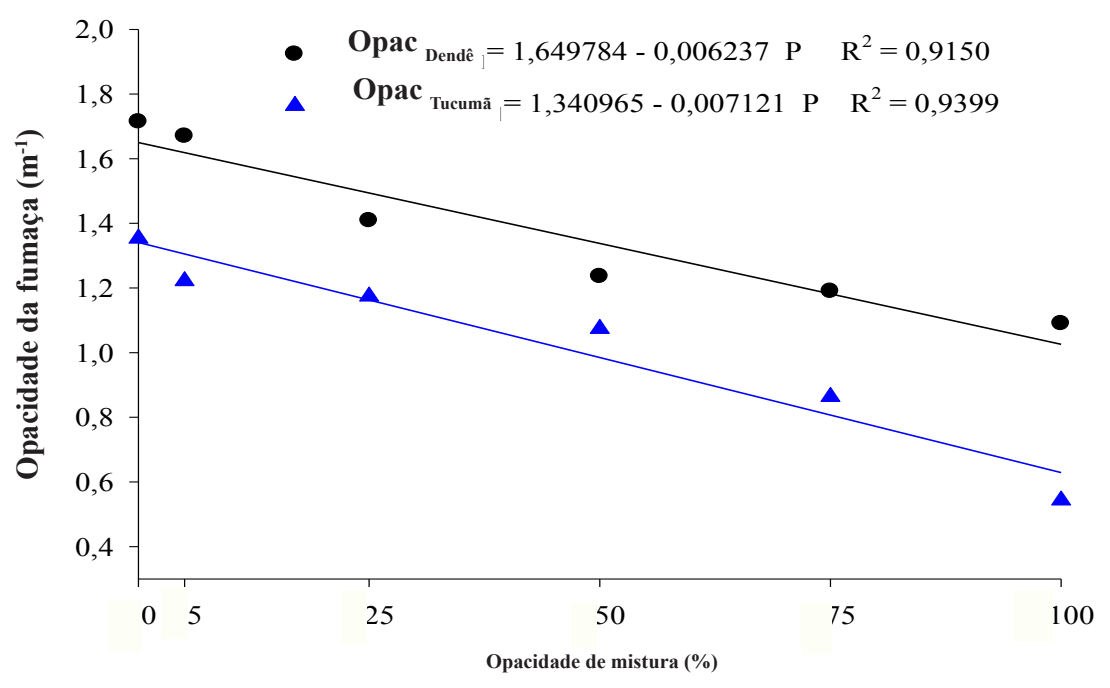

Figura 2. Modelo de regressão para opacidade da fumaça em função da proporção de mistura de biodiesel de dendê, mamona e tucumã no diesel. 
Quadro 3. Síntese do desdobramento da interação tipo e proporção de biodiesel para a variável opacidade da fumaça $\left(\mathrm{m}^{-1}\right)$

\begin{tabular}{ccccccc}
\hline & \multicolumn{5}{c}{ Proporção de Biodiesel } \\
\cline { 2 - 7 } Tipo de Biodiesel & $\mathbf{0}$ & $\mathbf{5}$ & $\mathbf{2 5}$ & $\mathbf{5 0}$ & $\mathbf{7 5}$ & $\mathbf{1 0 0}$ \\
\hline Dendê & $1,71 \mathrm{Aa}$ & $1,67 \mathrm{Aa}$ & $1,40 \mathrm{Ab}$ & $1,23 \mathrm{Ac}$ & $1,19 \mathrm{Ac}$ & $1,09 \mathrm{Ad}$ \\
Tucumã & $1,35 \mathrm{Ba}$ & $1,22 \mathrm{Bb}$ & $1,17 \mathrm{Bb}$ & $1,07 \mathrm{Bc}$ & $0,86 \mathrm{Bd}$ & $0,54 \mathrm{Be}$ \\
\hline
\end{tabular}

Médias seguidas de mesma letra maiúscula na coluna e minúscula na linha não deferem entre si, pelo teste de Tukey a $5 \%$ de probabilidade.

\section{CONCLUSÕES}

Com base nos resultados obtidos, pode-se concluir que:

- O incremento de biodiesel até o limite de $50 \%$ não altera significativamente o consumo específico do trator para as condições do ensaio;

- O uso de $100 \%$ de biodiesel no trator não limitou o funcionamento do mesmo, entretanto, aumentou o consumo específico em 23,51\%, sendo isso justificado, principalmente, pelo menor poder calorífico do B100 em relação ao diesel de petróleo;

- A opacidade da fumaça tem uma redução de $47,0 \%$ quando o trator utilizou biodiesel B100 como combustível; e

- A opacidade da fumaça do motor é influenciada pelo tipo de biodiesel, com melhores resultados para o biodiesel de tucumã, opacidade da fumaça reduziu à medida que aumentou a proporção de mistura no diesel.

\section{REFERÊNCIAS BIBLIOGRÁFICAS}

ANDREOLI, I.; CENTURION, J.F. Levantamento detalhado dos solos da Faculdade de Ciências Agrárias e Veterinárias de Jaboticabal. In: CONGRESSO BRASILEIRO DE CIÊNCIA DO SOLO, 27, 1999, Brasília. Anais... Brasília: Sociedade Brasileira de Ciência do Solo, 1999. 32p. (TO25-3 CD-ROM).

BANZATTO, D.A.; KRONKA, S.N. Experimentação agrícola. $4^{\mathrm{a}}$ ed. Jaboticabal:
FUNEP, 2006. 237p.

BRASIL. Resolução ANP $n^{\circ}$ 4, de 2 de fevereiro de 2010. Dispõe sobre a introdução do biodiesel na matriz energética brasileira. Diário Oficial da República Federativa do Brasil, Brasília, DF, 3 fev. 2010. Disponível em: $<$ https://legislacao.planalto. gov.br/ legislacao.nsf> Acesso em: março. 2012.

CAMARA, F.T. Biodiesel de dendê em trator agrícola: desempenho em função do tempo de armazenamento e da proporção de mistura na operação de preparo do solo. 2009. 92f. Tese (Doutorado em Agronomia) - Universidade Estadual Paulista, Faculdade de Ciências Agrárias e Veterinárias, Jaboticabal, 2009.

CAMARA, F.T.; LOPES, A.; DABDOUB, M.J.; BORSATTO, E.A.; ZANOTTO, R.P.C.; REIS, G.N. Biodiesel de dendê: opacidade da fumaça de um trator agrícola. In: CONGRESSO BRASILEIRO DE PLANTAS OLEAGINOSAS, ÓLEOS, GORDURAS E BIODIESEL"BIODIESEL: COMBUSTÍVEL ECOLÓGICO", 4., 2007, Varginha. Anais... Lavras: UFLA, 2007. p.586-591.

CASTELLANELLI, M.; SOUZA, S.N.M.; SILVA, S.L.; KAILER, E.K. Desempenho de motor ciclo diesel em bancada dinamométrica utilizando misturas diesel/biodiesel. Engenharia Agrícola, Jaboticabal, v.28, n.1, p.145-53, jan./mar.,2008.

CASTRO, J.C.; SILVA, L.P.; BARRETO, A.C. Produção sustentável de biodiesel a partir de oleaginosas amazônicas em comunidades isoladas. In: CONGRESSO DA REDE BRASILEIRA DE TECNOLOGIA DE BIODIESEL, 1, 2006, 
Brasília/DF. Anais..., v. 1, p. 285-289, 2006.

CONTROLE do aquecimento ainda tem custo baixo. Jornal Estado de Minas, Belo Horizonte, $1^{\circ}$ maio 2007. Ciência, p. 18. c, 1- 3.

CORRÊA, I.M.; MAZIERO, J.V.G.; ÚNGARO, M.R.; BERNARDI, J.A.; STORINO, M. Desempenho de motor diesel com misturas de biodiesel de óleo de girassol. Ciência e Agrotecnologia, Lavras, v.32, n.3, p. 923-928, mai.jun., 2008.

DABDOUB, M.J.; BRONZEL, J.L. RAPIN, M.A. Biodiesel: visão crítica do status atual e perspectivas na academia e na indústria. Química Nova, São Paulo, v.32, n.3, p.776-792, abril. 2009.

FIGLIUOLO, R.; NUNOMURA, S.M.; SILVA, J.D.; CASTRO, J.C. Prospecção para o uso adequado e sustentável de sementes oleaginosas na produção de biodiesel na amazônia, 2004, Salvador. Resumos da XXVII Reunião Anual da SBQ. Salvador: SBQ. 2004.

FIGLIUOLO, R.; SILVA, J.D.; COSTA, M.S.T.A. Produção de Biodiesel na Cadeia Produtiva e Sustentável do Tucumã do Amazonas (Astrocaryum aculeatum G.F.W. Mayer) (Arecácea, palmeira). In: CONGRESSO DA REDE BRASILEIRA DE TECNOLOGIA DE BIODIESEL, 2, 2007, Brasília/DF. Anais..., p. 73-73.

GROTTA, D.C.C.; LOPES, A.; FURLANI, C.E.A.; SILVA, R.P.; REIS, G.N.; CORTEZ, J. W. Biodiesel etílico de óleo residual de soja: desempenho de um trator agrícola na operação de gradagem. Acta Scientiarum Technology, Maringá, v.30, n.2, p.135-138, março. 2008.

LÔBO, I.P.; FERREIRA, S.L.C. Biodiesel: Parâmetros de qualidade e métodos analíticos. Química Nova, São Paulo, v.32, n.6, p.1596-1608, julho. 2009.

LOPES, A. Biodiesel em trator agrícola: Desempenho e Opacidade. 2006, 158f. Tese (Livre Docência em Agronomia) - Faculdade de
Ciências Agrárias e Veterinárias de Jaboticabal, Universidade Estadual Paulista, Jaboticabal, 2006.

LOPES, A.; CAMARA, F.T.; TABILE, R.A.; SILVA, R.P.; FURLANI, C.E.A.; BARBOSA, A.L.P.B.F. Diesel S500 x Interior: opacidade da fumaça de tratar agrícola- Parte I. In: CONGRESSO BRASILEIRO DE PLANTAS OLEAGINOSAS, ÓLEOS, GORDURAS E BIODIESEL"BIODIESEL: COMBUSTÍVEL ECOLÓGICO", 4., 2007, Varginha. Anais... Lavras: UFLA, 2007. p.625-628.

LOPES, A.; SILVA, R.P.; FURLANI, C.E.A.; CASTRO NETO, P.; FRAGA, A.C.; REIS, G. N.; NAGAOKA, A.K. Potencialidades do Biodiesel no Brasil. In: CONGRESSO BRASILEIRO DE PLANTAS OLEAGINOSAS, ÓLEOS VEGETAIS E BIODIESEL, 1., Varginha, 2004. Anais... Varginha: UFLA, 2004. 1 CD-ROM.

MURUGESAN, A.; UMARANI, C.; SUBRAMANIAN, R.; NEDUNCHEZHIAN, N. Bio-diesel as an alternative fuel for diesel engines. Renewable and Sustainable Energy Reviews, Uttaranchal, v.13, n.3, p. 653-662, abril. 2009.

NASCIMENTO, J.E.; DALLAN, J.C.F. O consumo e a produção de biodiesel e a necessidade de ações mitigadoras de efeito estufa. In: CONGRESSO BRASILEIRO DE PLANTAS OLEAGINOSAS, ÓLEOS, GORDURAS E BIODIESEL"BIODIESEL: COMBUSTÍVEL ECOLÓGICO", 4., 2007, Varginha. Anais... Lavras: UFLA, 2007. p.73-83.

PARENTE, E.J.S. Biodiesel: Uma aventura tecnológica em um país engraçado. Fortaleza: Universidade Federal do Ceará. Tecbio, 2003. 68p.

PEREIRA, D.P.; DANTAS, A.A.A. Potencial de redução da poluição do ar causada pelas emissões de motores diesel, com a implementação do biodiesel. In: CONGRESSO BRASILEIRO DE PLANTAS OLEAGINOSAS, ÓLEOS, GORDURAS E BIODIESEL- "BIODIESEL: COMBUSTÍVEL ECOLÓGICO”, 4., 2007, Varginha. Anais... Lavras: UFLA, 2007. p. 383-397. 
PERES, S.; SCHULER, A.; ALMEIDA, C.H.T.; SOARES, M.B.; CAMPOS, R.; LUCENA, A. Caracterização e Determinação do Poder Calorífico e do Número de Cetano de Vários Tipos de Biodiesel Através da Cromatografia In: CONGRESSO DA REDE BRASILEIRA DE TECNOLOGIA DE BIODIESEL, 2., 2007, Brasília. Anais... Brasília: SETEC/MCT/ABIPTI, 2007. 1 CD-ROM.

PIMENTEL, M.F.; RIBEIRO, G.M.G.S.; CRUZ, R.S.; STRAGEVITCH, L.; PACHECO FILHO, J.G.A.; TEIXEIRA, L.S.G. Determination of biodiesel content when blended with mineral diesel fuel using infrared spectroscopy and multivariate calibration. Microchemical Journal, v. 82, p.201206, abril. 2006.

PINTO, A.C.; GUARIEIRO, L.L.N.; REZENDE, M.J.C.; RIBEIRO, N.M.; TORRES E.A.; LOPES, W.A.; PERREIRA, P.A.P.; ANDRADE, J.B. Biodiesel: An Overview. Journal Brazilian Chemists Society, v.16, n.6B, p.1313-1330, nov./ dez., 2005.
SANTANA, A.C.; SANTANA, A.L.; FILGUEIRAS, G.C. Identificação e análise de arranjos produtivos locais na BR-163: 2002-2003 Amazônia: ciência e desenvolvimento. Belém: Banco da Amazônia. - v.1, n.1 (jul./dez. 2005). p.97-119.

SUAREZ, P.A.Z.; MENEGHETTI, S.M.P. $70^{\circ}$ Aniversário do biodiesel em 2007: evolução histórica e situação atual no Brasil. Química Nova, São Paulo, v.30, n.8, p.2068-2071, 2007.

TABILE, R.A.; LOPES, A.; DABDOUB, M.J.; CAMARA, F.T.; FURLANI, C.E.A.; SILVA, R.P. Biodiesel de mamona no diesel interior e metropolitano em trator agrícola. Engenharia Agrícola, Jaboticabal, v.29, n.3, p.412-423, jul./ set., 2009.

TECNOMOTOR, Tecnomotor Eletrônica do Brasil Ltda., OPACER TM 133 Opacímetro de amostragem. Manual de Operação. São Carlos, 2010. Ed. 07/01, 26p. 